\title{
Omics-based microbiome analysis in microbial ecology: from sequences to information
}

\author{
Jang-Cheon Cho \\ Department of Biological Sciences and Bioengineering, Inha University, \\ Incheon 22212, Republic of Korea
}

\begin{abstract}
Microbial ecology is the study of microorganisms present in nature. It particularly focuses on microbial interactions with any biota and with surrounding environments. Microbial ecology is entering its golden age with innovative multi-omics methods triggered by next-generation sequencing technologies. However, the extraction of ecologically relevant information from ever-increasing omics data remains one of the most challenging tasks in microbial ecology. This special issue includes 11 review articles that provide an overview of the state of the art of omics-based approaches in the field of microbial ecology, with particular emphasis on the interpretation of omics data, environmental pollution tracking, interactions in microbiomes, and viral ecology.
\end{abstract}

Keywords: omics, microbiome, source tracking, microbial interaction, viral ecology

\section{Introduction}

Microbial ecology covers all aspects of microbial theory and methods to explain the vital role of microorganisms present in natural or built environments. It encompasses studies on biogeochemical cycles, community structure and function, microbial interactions, and environmental pollution due to anthropogenic activities. Studies on the microbial community structure based on 16S rRNA gene sequences without cultivation since the late 1980s (Pace et al., 1985; Giovannoni et al., 1990) have revealed enormous diversity of uncultured microorganisms in nature. Subsequent environmental genomics research based on bacterial artificial chromosome clones (Béjà et al., 2000) has unveiled hidden metabolic functions of the microbiome, such as rhodopsin-based phototrophy and anaerobic methane oxidation.

At present, microbial ecology can be practically referred to as the study of the microbiome. The microbiome comprises "microbiota" (prokaryotic or eukaryotic microorganisms) and their "theatre of activities" (structural elements, e.g., mac-

${ }^{*}$ For correspondence. E-mail: chojc@inha.ac.kr; Tel.: +82-32-860-7711; Fax: +82-32-232-0541

Copyright (C) 2021, The Microbiological Society of Korea romolecules, and mobile genetic elements, e.g., viruses, microbial metabolites, and environmental conditions) (Berg et al., 2020), which are particularly assessed by multi-omics approaches. Omics technologies, including but not limited to (meta)genomics, (meta)transcriptomics, (meta)proteomics, metabolomics, epigenomics, single-cell genomics, and culturomics, which are powered by high-throughput sequencing technologies and bioinformatics, have provided detailed information on in situ microbial activities and on the critical roles of microorganisms in modulating the host metabolism. However, despite the tremendous amount of multi-omics data accumulated to understand the ecological roles of microorganisms, ranging from those in natural habitats to living holobionts, unraveling the mechanisms of microbial communities in influencing ecosystem functioning remains challenging.

This special issue introduces the recent advances in omicsbased approaches from selected fields of microbial ecology to gain insights into how to extract meaningful ecological information from the sea of omics data. It includes comprehensive reviews of the latest achievements and limitations in the following omics research areas: methodologies in the interpretation of metagenomes (Gwak et al., 2021), methylomes (Seong et al., 2021), and phenomes generated by Raman spectroscopy (Hong et al., 2021); tracking of antibiotic resistome (Lee et al., 2021) and microbial pollution sources (Raza et al., 2021); assessment of microbial interactions in the plant microbiome (Choi et al., 2021), human gut microbiome (Whon et al., 2021), and ammonium-oxidizing archaea (AOA) (Kim et al., 2021); and study of viral ecology involving aquatic virome analyses (Moon and Cho, 2021), roles of viral circular RNA (circRNA) (Lou et al., 2021), and severe acute respiratory syndrome coronavirus 2 (SARS$\mathrm{CoV}-2)$ from the one-health approach (Na et al., 2021).

\section{Interpretation of omics data}

In 2020, Journal of Microbiology published a special issue $(\mathrm{Na}, 2020)$ covering various protocols applied to learn computational methods, including microbiome analyses (Jo et al., 2020; Kim et al., 2020; Namkung, 2020), Ribo-Seq analyses (Choe et al., 2020), metabolic engineering (Jeon and Kim, 2020), and drug discovery (Shaker et al., 2020). To introduce the latest achievements not covered in that special issue, we have provided three reviews that may provide insights on how to interpret the data obtained from metagenomes, me- 
thylomes, and phenomes. In this special issue, Gwak et al. (2021) provides a comprehensive review of computational approaches for the investigation of metagenome data, focusing on the detailed process, which includes the assembly of metagenomic reads, generation of metagenome-assembled genomes, taxonomic and functional profiling of metagenome reads, and prediction of metabolomic profiles from metagenomes. Detailed procedures and software, including the recently developed deep learning-based Seq2Species (Busia et al., 2019) and DeepMicrobes (Liang et al., 2020), which can be practically applied to metagenomic data analyses, are summarized. The limitations of these approaches are also introduced.

Of the diverse omics methodologies, methylome analysis can be performed in a high-throughput manner as longread sequencing technologies, such as Pacific Biosciences' single-molecule real-time sequencing and Oxford Nanopore Technologies' nanopore sequencing platforms, have recently been developed (van Dijk et al., 2018; Logsdon et al., 2020). Studies on DNA methylation have primarily been conducted for eukaryotic systems; however, methylation is also important for prokaryotic systems. Hence, Seong et al. (2021) introduces diverse methods for analyzing bacterial methylomes and the implication of methylome in bacterial physiology and defense mechanism. At present, most bacterial methylome analyses focus on single strain-level bacterial physiology related to the host defense system, cell cycle, gene expression, and virulence. As bacterial epigenetic regulation is mainly mediated by DNA methylation, analyzing the methylome pattern in metagenomic sequencing data obtained through long-read sequencing technologies will reveal the role and process of epigenetic regulation in natural microbial populations.

While many omics-based approaches have used molecular tools for nucleic acid and protein analyses, phenotype-based omics approaches lag behind because of the lack of highthroughput phenome analysis methods. As one of the phenome analysis tools, Hong et al. (2021) provides a comprehensive overview of the methodology of Raman spectroscopy and introduces recent advances in the application of Raman spectroscopy to the field of microbial ecology. They have concluded that single-cell Raman spectrum analysis will be widely used as a next-generation phenotyping tool and applied to natural ecosystems for understanding microbial activity and function because it enables the noninvasive labeling and detection of single cells, determination of phenotypes by intracellular materials, and cellular activity-based profiling.

\section{Microbial source tracking and antibiotic resistome}

We are not only in the omics era but also in the Anthropocene (Waters et al., 2016), a new geological time period. Human activities adversely affect the environmental quality by introducing various pollutants into the ecosystem and subsequently causing environmental pollution. As microorganisms rapidly respond to the changing environment, they have been widely used as indicators or cleaners of environmental pollution. As a tool for assessing environmental pol- lution and tracking the sources of fecal pollution, microbial source tracking has become more important in the Anthropocene and has moved from traditional culture-dependent approaches to culture-independent approaches based on various omics technologies. In this special issue, Raza et al. (2021) addresses metagenomics and other recently developed sequencing technologies in light of microbial source tracking. Metagenomics-based microbial source tracking can rapidly replace microbial community-based source tracking methods by identifying host-specific fecal markers and their association with the environment because metagenomes cover all the genetic materials in a given environment.

Apart from fecal pollution, antibiotics and antibiotic resistance genes (ARGs) are regarded as environmental pollutants or intrinsic resistome. The emergence of antibiotic resistant bacteria through the acquisition of ARGs with intrinsic mechanisms or horizontal transfers is threatening our lives and is becoming a topic of great interest in the onehealth approach. As ARGs are widely distributed in microbiomes, viromes, and mobilomes even in pristine ecosystems, the natural environment is considered a reservoir of ARGs, making metaomics-based approaches prerequisite in ARG research. In this special issue, Lee et al. (2021) introduces up-to-date bioinformatic methods for analyzing antibiotic resistomes from whole genomes and metagenomic data. This review focuses on the detailed workflow procedures, software tools, and data resources that are implemented in ARG studies. On reading this article, readers will be able to catalogue the known ARGs from genomes and metagenomes, quantify the genes, and gain an insight into the evolution and dissemination of ARGs.

\section{Holobionts and microbial interactions}

The holobiont (i.e., a host and its microbiota) concept is becoming one of the major biological frameworks to explain intimate interactions between a host and its associated microbial communities. Symbiotic microorganisms or microorganisms associating with their hosts are even regarded as biological organs in animals and plants, affecting the biology, ecology, and evolution of the hosts. The microbiomes of plants and animals consists of all kinds of microorganisms, including bacteria, archaea, fungi, and algae, and collective information about microbial genomes, mobile genetic elements (including viruses), and microbial metabolites. Given that most symbiotic microorganisms are not maintained as axenic cultures outside their hosts, culture-independent metaomics technologies are a promising option for studying holobionts in a holistic view. The plant microbiome and animal gut microbiome are good model systems to understand the holobiont view of ecology and evolution because plants accept their microbial partners living in them or in soil as a compartment of their body and animals treat the gut microbiome as another organ. In this context, this special issue has invited two reviews presenting the recent advances in plant microbiome and gut microbiome research. In their review, Choi et al. (2021) encompasses an overview of the plant microbiome structure from the rhizosphere to the phyllosphere, microbiome function related to plant physiology, and research 
trends in the synthetic microbial community mimicking the plant microbiome to study the microbiome with a reductionist view. Moreover, Whon et al. (2021) have summarized recent gut microbiome research employing diverse metaomics approaches, discussed the advantages and disadvantages of these omics methods, and introduced the recent technological advances in gut microbiome research, including adaptive immune receptor repertoire sequencing, culturomics, and machine learning.

In this special issue, Kim et al. (2021) provides a comprehensive review of the roles of AOA in interactions with niche-sharing organisms and surrounding environments. AOA belonging to the phylum Thaumarchaeota are abundant and ubiquitous, particularly in meso- and bathypelagic ocean and soil environments, and show ammonium-oxidizing chemolithotrophy. Therefore, major research interests have been focused on their diversity and activities in diverse ecosystems; however, the interactions between AOA and other cohabiting bacteria, viruses, and higher organisms have been of lesser interest. Kim et al. (2021) have summarized the intimate interactions between AOA and all kinds of life forms, such as viruses, heterotrophic bacteria, ammonium-oxidizing bacteria, nitrite-oxidizing bacteria, protists, plants, and animals (sponge). However, the scarcity of cultured representatives of AOA hinders in-depth and comprehensive research on AOA in order to elucidate their ecological roles in biogeochemical cycling and interactions during this ecological process. Therefore, the cultivation of more AOA strains and accumulation of experimental evidence using the cultures would be important to elucidate the hidden roles of AOA in ecosystem functioning.

\section{Viral ecology}

Viruses are the smallest biological entities that can infect all known life forms on Earth and known to be most abundantly present in diverse environments. As viruses cannot replicate without their hosts, the biology of viruses is directly coupled to their ecology. Viral ecology includes the ecology of viruses infecting microorganisms (bacteria, archaea, and protists) and those infecting higher eukaryotes (plants and animals). As the number of bacteriophages and archaeal viruses in natural environments is more than 10 times higher than the number of prokaryotes, the ecology of microbial viruses has been studied as a discipline of microbial ecology to identify their ecological roles in carbon and material cycling, gene transfer, and reserving novel genetic repertoire. In contrast, the ecology of viruses infecting higher eukaryotes has focused on their etiological importance, such as host interaction, host specificity and range, types of transmission cycles, and transmission vehicles and vectors. As most bacteria and archaea in the ecosystem are not yet cultivated and as viruses that have the potential to infect animals, including humans, are undiscovered or rapidly evolving, viral assemblages (viromes) are considered reservoirs of novel genetic materials or functions.

Given the importance of viruses as ecosystem designers, genetic resources, and etiological agents, this special issue has invited three reviews describing the aquatic phage ecology based on multi-omics approaches, roles of host- and virusderived circular RNAs (circRNAs) during infection, and ecology of SARS-CoV-2) from a one-health perspective. Moon and Cho (2021) presents the recent trends in aquatic virome studies, with a particular focus on culturomics and metaviromics. In their review, they have described the detailed procedures for culture-dependent viral studies and viral metagenome analyses, approaches for predicting host-phage system in viromes, and experimental approaches for predicting phage-host interactions. Lou et al. (2021) introduces the classification, production, and functions of circRNAs in transcriptome analyses; roles of circRNAs during the process of viral infection; and use of circRNAs as biomarkers in the diagnosis of viral infection. $\mathrm{Na}$ et al. (2021) provides a comprehensive review of SARS-CoV-2, the virus that causes coronavirus disease 2019 (COVID-19) and continues to afflict humankind. They have provided the detailed phylogeny, mutation, and molecular epidemiology of SARS-CoV-2 and compared its pathological characteristics with those of other animal coronaviruses. As viruses that peacefully co-exist with their hosts find a way to enter new hosts that are highly populated and cause a severe outbreak, understanding the behaviors of the viruses interacting with their hosts is fundamental to control viral outbreaks. Therefore, $\mathrm{Na}$ et al. (2021) have discussed the ecology of SARS-CoV-2 in detail in the view of potential wildlife hosts and the zoonotic origin of this emerging virus.

Collectively, the 11 review articles provided in this special issue highlight the power of omics tools to unravel the infinite roles of infinite small microorganisms in natural environments and living systems. It is noteworthy that this special issue covers only the tip of the iceberg of the vast majority of microbiome studies in the field of microbial ecology. Many of the contributors to this series recognize machine learning and deep-learning technologies as promising tools to investigate countless data generated by omics approaches. Therefore, we must be prepared for facing the incoming innovative waves of this omics era.

\section{Acknowledgments}

This study was supported by the Basic Research Program through the National Research Foundation (NRF) funded by the Ministry of Sciences and ICT (NRF-2019R1A2B5B02070538).

\section{Conflict of Interest}

I have no conflicts of interest to report.

\section{References}

Béjà, O., Aravind, L., Koonin, E.V., Suzuki, M.T., Hadd, A., Nguyen, L.P., Jovanovich, S.B., Gates, C.M., Feldman, R.A., Spudich, J.L. et al. 2000. Bacterial rhodopsin: evidence for a new type of phototrophy in the sea. Science 289, 1902-1906.

Berg, G., Rybakova, D., Fischer, D., Cernava, T., Vergès, M.C.C., Charles, T., Chen, X., Cocolin, L., Eversole, K., Corral, G.H., et al. 
2020. Microbiome definition revisited: old concepts and new challenges. Microbiome 8, 103.

Busia, A., Dahl, G.E., Fannjiang, C., Alexander, D.H., Dorfman, E., Poplin, R., McLean, C.Y., Chang, P.C., and DePristo, M. 2019. A deep learning approach to pattern recognition for short DNA sequences. bioRxiv 353474. doi: https://doi.org/10.1101/353474.

Choe, D., Palsson, B., and Cho, B.K. 2020. STATR: A simple analysis pipeline of Ribo-Seq in bacteria. J. Microbiol. 58, 217-226.

Choi, K., Khan, R., and Lee, S.W. 2021. Dissection of plant microbiota and plant-microbiome interactions. J. Microbiol. 59, 281291.

Giovannoni, S.J., Britschgi, T.B., Moyer, C.L., and Field, K.G. 1990. Genetic diversity in Sargasso Sea bacterioplankton. Nature 345, 60-63.

Gwak, H.J., Lee, S.J., and Rho, M. 2021. Application of computational approaches to analyze metagenomic data. J. Microbiol. 59, 233 241.

Hong, J.K., Kim, S.B., Lyou, E.S., and Lee, T.K. 2021. Microbial phenomics linking the phenotype to function: The potential of Raman spectroscopy. J. Microbiol. 59, 249-258.

Jeon, J. and Kim, H.U. 2020. Setup of a scientific computing environment for computational biology: Simulation of a genome-scale metabolic model of Escherichia coli as an example. J. Microbiol. 58, 227-234.

Jo, J., Oh, J., and Park, C. 2020. Microbial community analysis using high-throughput sequencing technology: a beginner's guide for microbiologists. J. Microbiol. 58, 176-192.

Kim, J.G., Gazi, K.S., Awala, S.I., Jung, M.Y., and Rhee, S.K. 2021. Ammonia-oxidizing archaea in biological interactions. J. Microbiol. 59, 298-310.

Kim, H., Kim, S., and Jung, S. 2020. Instruction of microbiome taxonomic profiling based on $16 \mathrm{~S}$ rRNA sequencing. J. Microbiol. 58, 193-205.

Lee, K., Kim, D.W., and Cha, C.J. 2021. Overview of bioinformatic methods for analysis of antibiotic resistome from genome and metagenome data. J. Microbiol. 59, 270-280.

Liang, Q., Bible, P.W., Liu, Y., Zou, B., and Wei, L. 2020. DeepMicrobes: taxonomic classification for metagenomics with deep learning. NAR Genom. Bioinform. 2, lqaa009.
Logsdon, G.A., Vollger, M.R., and Eichler, E.E. 2020. Long-read human genome sequencing and its applications. Nat. Rev. Genet. 21, 597-614.

Lou, Z., Zhou, R., Su, Y., Liu, C., Ruan, W., Jeon, C.O., Han, X., Lin, C., and Jia, B. 2021. Minor and major circRNAs in virus and host genomes. J. Microbiol. 59, 324-331.

Moon, K. and Cho, J.C. 2021. Metaviromics coupled with phagehost identification to open the viral 'black box.' J. Microbiol. 59, 311-323.

Na, D. 2020. User guides for biologists to learn computational methods. J. Microbiol. 58, 173-175.

Na, W., Moon, H., and Song, D. 2021. A comprehensive review of SARS-CoV-2 genetic mutations and lessons from animal coronavirus recombination in one health perspective. J. Microbiol. 59, 332-340.

Namkung, J. 2020. Machine learning methods for microbiome studies. J. Microbiol. 58, 206-216.

Pace, N.R., Stahl, D.A., Lane, D.J., and Olsen, G.J. 1985. Analyzing natural microbial populations by rRNA sequences. ASM News 51, 4-12.

Raza, S., Kim, J., Sadowsky, M.J., and Unno, T. 2021. Microbial source tracking using metagenomics and other new technologies. $J$. Microbiol. 59, 259-269.

Seong, H.J., Han, S.W., and Sul, W.J. 2021. Prokaryotic DNA methylation and its functional roles. J. Microbiol. 59, 242-248.

Shaker, B., Yu, M.S., Lee, J., Lee, Y., Jung, C., and Na, D. 2020. User guide for the discovery of potential drugs via protein structure prediction and ligand docking simulation. J. Microbiol. 58, 235244.

van Dijk, E.L., Jaszczyszyn, Y., Naquin, D., and Thermes, C. 2018. The third revolution in sequencing technology. Trends Genet. 34, 666-681.

Waters, C.N., Zalasiewicz, J., Summerhayes, C., Barnosky, A.D., Poirier, C., Gałuszka, A., Cearreta, A., Edgeworth, M., Ellis, E.C., Ellis, M., et al. 2016. The Anthropocene is functionally and stratigraphically distinct from the Holocene. Science 351, aad2622.

Whon, T.W., Shin, N.R., Kim, J.Y., and Roh, S.W. 2021. Omics in gut microbiome analysis. J. Microbiol. 59, 292-297. 